\title{
Comparisons of High Order Hybrid Schemes Using a Parameter-free Discontinuity Detector
}

\author{
Yiqing Shen*1, Gecheng Zha ${ }^{\dagger 2}$, Guowei Yang ${ }^{1}$, and Zhi Gao ${ }^{1}$ \\ ${ }^{1}$ LHD, Institute of Mechanics, Chineses Academy of Sciences, \\ 100190, Beijing, China \\ ${ }^{2}$ Dept. of Mechanical and Aerospace Engineering, University of Miami, \\ Coral Gables, Florida 33124, USA
}

\begin{abstract}
In order to simulate accurately the interaction of shock wave/complex flows, hybrid schemes combining the compact scheme and weighted essentially non-oscillatory(WENO) scheme are beneficial, especially the high order low diffusion schemes used in smooth regions. There are two key issues, one is how to detect the discontinuity, the other is how to choose the high order low diffusion scheme to ensure the accuracy, robustness and efficiency. In this paper, several high order schemes are combined with the fifth order WENO scheme by using the discontinuity detector proposed by Shen and Zha(Y. Shen, G.Zha, J. Compu. Phys., 2011, 230:4419-4436) and compared numerically.
\end{abstract}

\section{Introduction}

The numerical schemes for direct numerical simulation (DNS) and large eddy simulation (LES) of interaction of shock waves/complex flows need to resolve both shock waves and fine flow structures. Since weighted essentially non-oscillatory (WENO) schemes tend to have uniform higher order accuracy in smooth region and keep the essentially non-oscillatory properties up to the vicinity of shock waves, they are widely used in simulation of flows with discontinuities such as shock waves and contact surfaces[1]. However, even though the order of accuracy for WENO schemes can be designed to be arbitrarily high[2,3], the resolution of short waves of WENO schemes in smooth regions tends to be more diffusive than the central differencing schemes with an equivalent order of accuracy.

The most accurate method to simulate wave dominated problems is the spectral method[4], but it is limited to simple geometries with generally periodic boundary conditions. Due to compact schemes' spectral-like resolution and their flexibility, compact schemes[5] have attracted a lot of attention and have been widely used for DNS and LES of turbulence flows[6, 7, 8, 9, 10, 11]. A drawback of compact schemes is that they will generate Gibbs-like oscillations around shock waves or large gradients.

In recent years, there are many efforts to make compact schemes possess shock-capturing capability. Cockburn and Shu[12] develop the nonlinear stable compact schemes using the TVDM(total variation diminishing in the means) property. The schemes require an implicit symmetric matrix and a reconstruction from the mean variable obtained by TVDM compact schemes. Ravichandran[13] improves this type of schemes with a class of the compact upwind schemes developed without the limitation of a symmetric

* yqshen@imech.ac.cn

$\dagger$ AIAA Senior Member, gzha@miami.edu 
matrix. Tu and Yuan[14] construct a fifth-order shock-capturing compact upwind scheme by using a characteristic-based flux splitting limited method.

Adams and Shariff[15] propose a hybrid compact-ENO scheme for shock-turbulence interaction problems. Following the same basic approach, Pirozzoli[16] derives a conservative hybrid compact-WENO scheme. Ren et al.[17] present a fifth-order conservative hybrid compact-WENO scheme for shock-capturing calculation, which is constructed through the weighted average of conservative compact scheme and WENO scheme. Zhou et al. [18] suggest a new family of high order compact upwind difference schemes, which are later made to have shock-capturing capability by combining them with WENO schemes.

Shen et al.[19] propose a finite compact scheme, which treats the discontinuity as the internal boundary and avoids the global dependence of the traditional compact schemes. Combined with the TVD of ENO limiters, a set of high resolution finite compact (FC) difference schemes with only bi-diagonal matrix inversion are constructed[19, 20].

Some of the above methods need to calculate the preliminary fluxes first by using a standard compact scheme[12, 13, 14, 15, 16]. This may result in contaminated (oscillatory) fluxes in the regions near discontinuities, and hence the compact fluxes will lose their high order accuracy in those regions. Some other methods using limiter functions will degrade the accuracy at extrema to first order[12, 13, 14, 19]. All the hybrid schemes $[12,15,16,17,18,19]$ introduce a free parameter to judge the flow gradient and to switch to the ENO/WENO schemes at discontinuities. Such parameters are usually problem dependent and hence lose their generality. The weighted compact nonlinear schemes proposed by Deng and Zhang[21] need to use more nodes than a standard compact scheme and hence lose the compactness. The same problem exists in the higher order extensions[2] of Deng and Zhang's method.

Artificial dissipation and compact filters are also introduced into compact schemes to help stabilize numerical solutions and reduce oscillations near discontinuities[11, 22, 23, 24, 25, 26, 27, 28]. Nonlinear characteristics-based(artificial compression method, ACM) filters is used to construct the low-dissipative high order shock-capturing scheme by Yee et al.[29] with a problem dependent parameter introduced. A WENO-type smoothness estimator is used in [30] as a sensor to switch between the high-order compact spatial filters and the ACM filters, for which a threshold parameter for the sensor is also needed.

For the WENO schemes first proposed by Liu et al.[31] and improved by Jiang and Shu[32], a small difference between the smoothness estimators can reduce the numerical accuracy. A function to decrease the weights sensitivity to the smoothness estimators variation is suggested by Shen and Zha[33] to improve the WENO scheme accuracy. In addition, when it is applied to practical flows, the original value of the parameter $\varepsilon$ used to construct the weight functions will generate oscillatory weights, which result in convergence and accuracy problems. An optimized $\varepsilon$ value of $10^{-2}$ is suggested by Shen et al.[34] to improve convergence and accuracy. On the other hand, Henrick et al.[35] point out that the original smoothness indicators of Jiang and Shu fail to improve the accuracy order of a WENO scheme at critical points, where the first derivatives are zero. A mapping function is proposed by Henrick et al.[35] to obtain the optimal order near critical points. Recently, Borges et al.[36] suggest to use the whole 5-points stencil to devise a smoothness indicator of higher order than the classical smoothness indicator proposed by Jiang and Shu[32]. Shen and Zha[37] apply these smoothness estimators to improve accuracy at transition points of a discontinuity.

Based on the results of Borges et al.[36], Shen and Zha[38] propose a lemma without introducing any free parameters to detect shock regions and develop a class of generalized finite compact difference schemes by using the lemma. In this paper, the high order low diffusion schemes, including the fourth order central scheme, the fifth order upstream central scheme, the fifth order upwind compact scheme, and the sixth order Pade scheme, are combined with the fifth order WENO scheme by using the lemma, and their performances are compared numerically. 


\section{The Numerical Algorithm}

For the hyperbolic conservation law in the form

$$
\frac{\partial u}{\partial t}+\frac{\partial f}{\partial x}=0
$$

the flux function $f(u)$ can be split into two parts as $f(u)=f^{+}(u)+f^{-}(u)$ with $d f^{+}(u) / d u \geq 0$ and $d f^{-}(u) / d u \leq 0$. The semi-discretization form of (1) can be written as

$$
\frac{d u_{i}(t)}{d t}=-\frac{1}{\Delta x}\left(h_{i+\frac{1}{2}}-h_{i-\frac{1}{2}}\right)
$$

where the numerical flux is $h_{i+1 / 2}=h_{i+1 / 2}^{+}+h_{i+1 / 2}^{-}$. In this paper, only the positive part $h_{i+1 / 2}^{+}$is described and the superscript " + " is dropped for simplicity. The $h_{i+1 / 2}^{-}$is evaluated following the symmetric rule about $x_{i+1 / 2}$.

\subsection{Weighted essentially non-oscillatory(WENO) Scheme}

The flux $h_{i+\frac{1}{2}}$ of the classical fifth-order WENO scheme $[32,36]$ is built through the convex combination of interpolated values $\hat{f}^{k}\left(x_{i+\frac{1}{2}}\right)(k=0,1,2)$, in which $\hat{f}^{k}(x)$ is the third degree interpolation polynomial on stencil $S_{k}^{3}=\left(x_{i+k-2}, x_{i+k-1}, x_{i+k}\right)$,

$$
h_{i+\frac{1}{2}}=\sum_{k=0}^{2} \omega_{k} \hat{f}^{k}\left(x_{i+\frac{1}{2}}\right)
$$

where

$$
\hat{f}^{k}\left(x_{i+\frac{1}{2}}\right)=\hat{f}_{i+\frac{1}{2}}^{k}=\sum_{j=0}^{2} c_{k j} f_{i-k+j}, \quad i=0, \cdots, N
$$

The weights $\omega_{k}$ are defined as

$$
\omega_{k}=\frac{\alpha_{k}}{\sum_{l=0}^{2} \alpha_{l}}, \quad \alpha_{k}=\frac{d_{k}}{\left(\beta_{k}+\varepsilon\right)^{p}}
$$

The smoothness indicators $\beta_{k}$ are given as[32]

$$
\beta_{k}=\sum_{l=1}^{2} \Delta x^{2 l-1} \int_{x_{i-\frac{1}{2}}}^{x_{i+\frac{1}{2}}}\left(\frac{d^{l}}{d x^{l}} \hat{f}^{k}(x)\right)^{2} d x
$$

Taylor expansion of (6) gives

$$
\left\{\begin{array}{l}
\beta_{0}=\frac{13}{12}\left(f_{i-2}-2 f_{i-1}+f_{i}\right)^{2}+\frac{1}{4}\left(f_{i-2}-4 f_{i-1}+3 f_{i}\right)^{2} \\
\beta_{1}=\frac{13}{12}\left(f_{i-1}-2 f_{i}+f_{i+1}\right)^{2}+\frac{1}{4}\left(f_{i-1}-f_{i+1}\right)^{2} \\
\beta_{2}=\frac{13}{12}\left(f_{i}-2 f_{i+1}+f_{i+2}\right)^{2}+\frac{1}{4}\left(3 f_{i}-4 f_{i+1}+f_{i+2}\right)^{2}
\end{array}\right.
$$

Henrick et al.[35] indicate that if $\beta_{k}$ satisfy $\beta_{k}=D\left(1+O\left(\Delta x^{s}\right)\right)$, the weights $\omega_{k}$ then satisfy $\omega_{k}=$ $d_{k}+O\left(\Delta x^{s}\right)$, where $D$ is some non-zero quantity independent of $k$. And the necessary and sufficient conditions for fifth-order convergence in (2) are given as[35]:

$$
\sum_{k=0}^{2} A_{k}\left(\omega_{k}^{+}-\omega_{k}^{-}\right)=O\left(\Delta x^{3}\right)
$$


Table 1: Coefficients $c_{k j}$ and $d_{k}$

\begin{tabular}{cccc|c}
\hline & \multicolumn{3}{c|}{$c_{k j}$} & $d_{k}$ \\
\hline $\mathrm{k}$ & $\mathrm{j}=0$ & $\mathrm{j}=1$ & $\mathrm{j}=2$ & \\
\hline 0 & $1 / 3$ & $-7 / 6$ & $11 / 6$ & $1 / 10$ \\
1 & $-1 / 6$ & $5 / 6$ & $1 / 3$ & $6 / 10$ \\
2 & $1 / 3$ & $5 / 6$ & $-1 / 6$ & $3 / 10$ \\
\hline
\end{tabular}

$$
\omega_{k}^{ \pm}-d_{k}=O\left(\Delta x^{2}\right)
$$

where $A_{0}=-\frac{1}{4} f_{j}^{\prime \prime \prime}, A_{1}=\frac{1}{12} f_{j}^{\prime \prime \prime}$, and $A_{2}=-\frac{1}{12} f_{j}^{\prime \prime \prime}$ are the coefficients of the Taylor series expansions of

$$
\hat{f}_{i \pm \frac{1}{2}}^{k}=h_{i \pm \frac{1}{2}}^{5 U C}+A_{k} \Delta x^{3}+O\left(\Delta x^{4}\right)
$$

where $h_{i \pm \frac{1}{2}}^{5 U C}=\sum_{k=0}^{2} d_{k} \hat{f}_{i \pm \frac{1}{2}}^{k}$ is the fifth order upstream central scheme.

A sufficient condition for fifth-order of convergence is given by[36]:

$$
\omega_{k}^{ \pm}-d_{k}=O\left(\Delta x^{3}\right)
$$

If $f_{i}^{\prime}=0$, Eq.(7) gives $\beta_{k}=D(1+O(\Delta x))$ and $\omega_{k}=d_{k}+O(\Delta x)$, this will degrade the convergence accuracy of the scheme. Shen et al [39] propose a step-by-step reconstruction method, in which two 4th order weighted fluxes obtained from 3rd ENO fluxes are used to construct 5th order WENO scheme. Henrick et al.[35] propose a mapping function to make the accuracy of $\omega_{k}$ closer to that of the ideal weights $d_{k}$.

Borges et al. [36] introduce the absolute difference between $\beta_{0}$ and $\beta_{2}$ to devise a new set of WENO weights that satisfies the necessary and sufficient conditions of fifth-order convergence. The new smoothness indicators $\beta_{k}^{z}$ defined by Borges et al. [36] are

$$
\beta_{k}^{z}=\frac{\beta_{k}+\varepsilon}{\beta_{k}+\tau_{5}+\varepsilon}, \quad k=0,1,2
$$

and the new WENO weights $\omega_{k}^{z}$ are

$$
\omega_{k}^{z}=\frac{\alpha_{k}^{z}}{\sum_{l=0}^{2} \alpha_{l}^{z}}, \quad \alpha_{k}^{z}=\frac{d_{k}}{\beta_{k}^{z}}=d_{k}\left(1+\left(\frac{\tau_{5}}{\beta_{k}+\varepsilon}\right)^{q}\right), \quad k=0,1,2
$$

where

$$
\tau_{5}=\left|\beta_{0}-\beta_{2}\right|
$$

The coefficients $c_{k j}$ and $d_{k}$ are list in Table 1. The parameter $\varepsilon$ is used to avoid the division by zero $\left(\varepsilon=10^{-6}\right.$ is used in [32] and $\varepsilon=10^{-40}$ is used in [36]), $p$ in (5) and $q$ in (13) are chosen to increase the difference of scales of distinct weights at non-smooth parts of the solution. As pointed out by Borges et al.[36], for a smooth function, increasing the value of $q$ in Eq. (13) decreases the correction of the WENO-Z weights to the ideal weights $d_{k}$, making the scheme closer to the optimal central scheme; on the other hand, increasing $q$ also decreases the relative importance of the discontinuous substencil and makes the scheme more dissipative. In this paper, $q=1$ is used.

\subsection{The important properties of $\tau_{5}$ and the lemma of discontinuity detection}

Taylor series expansions of $\beta_{k}$, Eq.(7), at $x_{i}$ are 


$$
\begin{aligned}
\beta_{0}=f_{i}^{\prime 2} \Delta x^{2}+\left(\frac{13}{12} f_{i}^{\prime \prime 2}-\frac{2}{3} f_{i}^{\prime} f_{i}^{\prime \prime \prime}\right) \Delta x^{4} & -\left(\frac{13}{6} f_{i}^{\prime \prime} f_{i}^{\prime \prime \prime}-\frac{1}{2} f_{i}^{\prime} f_{i}^{(4)}\right) \Delta x^{5} \\
& +\left(\frac{43}{36} f_{i}^{\prime \prime \prime 2}+\frac{91}{72} f_{i}^{\prime \prime} f_{i}^{(4)}-\frac{7}{30} f_{i}^{\prime} f_{i}^{(5)}\right) \Delta x^{6}+O\left(\Delta x^{7}\right) \\
\beta_{1}=f_{i}^{\prime 2} \Delta x^{2}+\left(\frac{13}{12} f_{i}^{\prime \prime 2}+\frac{1}{3} f_{i}^{\prime} f_{i}^{\prime \prime \prime}\right) \Delta x^{4} & +\left(\frac{1}{36} f_{i}^{\prime \prime \prime 3}+\frac{13}{72} f_{i}^{\prime \prime} f_{i}^{(4)}+\frac{1}{120} f_{i}^{\prime} f_{i}^{(5)}\right) \Delta x^{6}+O\left(\Delta x^{8}\right) \\
\beta_{2}=f_{i}^{\prime 2} \Delta x^{2}+\left(\frac{13}{12} f_{i}^{\prime \prime 2}-\frac{2}{3} f_{i}^{\prime} f_{i}^{\prime \prime \prime}\right) \Delta x^{4}+\left(\frac{13}{6} f_{i}^{\prime \prime} f_{i}^{\prime \prime \prime}-\frac{1}{2} f_{i}^{\prime} f_{i}^{(4)}\right) \Delta x^{5} & \\
& +\left(\frac{43}{36} f_{i}^{\prime \prime \prime 2}+\frac{91}{72} f_{i}^{\prime \prime} f_{i}^{(4)}-\frac{7}{30} f_{i}^{\prime} f_{i}^{(5)}\right) \Delta x^{6}+O\left(\Delta x^{7}\right)
\end{aligned}
$$

From Eqs. (14) and (15), it is easy to find that

$$
\tau_{5}=\left|\beta_{0}-\beta_{2}\right|=\frac{13}{3}\left|f_{i}^{\prime \prime} f_{i}^{\prime \prime \prime}\right| \Delta x^{5}+O\left(\Delta x^{7}\right)
$$

In fact, it can be proved that $\beta_{0}$ and $\beta_{2}$ have the same even order terms. And $\tau_{5}$ is at least one order higher than $\beta_{k}(k=0,1,2)$. For example, if $f_{i}^{\prime}=f_{i}^{\prime \prime}=0$ and $f_{i}^{\prime \prime \prime} \neq 0$, then $\beta_{k}=A_{k} \Delta x^{6}+O\left(\Delta x^{7}\right), A_{0}=A_{2}$ and $\tau_{5}=B \Delta x^{7}+O\left(\Delta x^{9}\right)$, and so on. $A_{k}$ and $B$ here are constants independent of $\Delta x$.

For completeness, the important properties of $\tau_{5}[36]$ are listed below:

(1) For a stencil $S^{5}=\left(x_{i-2}, x_{i-1}, \cdots, x_{i+2}\right)$, if $S^{5}$ does not contain discontinuities, then $\tau_{5}<<\beta_{k}$ for $k=0,1,2$;

(2) if the solution is continuous at some of the stencil $S_{k}^{3}$, but discontinuous in the whole stencil $S^{5}$, then for the smooth stencils, $\beta_{k}<<\tau_{5}$.

(3) $\tau_{5} \leq \max \left(\beta_{0}, \beta_{1}, \beta_{2}\right)$.

Following the above properties, Shen and Zha give the following lemma as the discontinuity detector[38]:

Lemma 1. If $\tau_{5}>\min \left(\beta_{0}, \beta_{1}, \beta_{2}\right)$, then $\mathbf{S}^{5}$ is a non-smooth stencil, which includes the function discontinuities and derivative discontinuities up to order $\mathbf{n}$ provided all the derivatives lower than order $\mathbf{n}$ are zero.

For example, if $f^{\prime}=0, f^{\prime \prime} \neq 0$, then $n=2$; if $f^{\prime}=0, f^{\prime \prime}=0$, and $f^{\prime \prime \prime} \neq 0, n=3$.

\subsection{The High Order Low Diffusion Hybrid Schemes}

In [38], a generalized finite compact difference is suggested by Shen and Zha as the following:

(1) A zone containing a discontinuity is detected by using Lemma 1, and the fluxes within this zone including at the zone boundaries are calculated by a WENO scheme.

(2) those smooth point(s) between two zones containing discontinuities(or between boundary point and discontinuous interface)are defined as a compact stencil, and a compact scheme is used to calculate the numerical fluxes on the compact stencil.

In this paper, the above algorithm is generalized by using different high order schemes to substitute the compact scheme used in step (2). The high order schemes compared in this paper include the fourth order central difference scheme, the fifth order upstream difference scheme, the fifth order upwind compact scheme and the sixth order Pade scheme.

Note: in step (2), the fluxes on the discontinuity zones obtained from step (1) are automatically used as the internal boundary fluxes.

Fig. 1 is a sketch showing this finite compact scheme. $M$ denotes the number of compact stencil divided by discontinuities. 


\subsection{Procedure of the High Order Low Diffusion Hybrid Schemes}

\section{BEGIN}

Step 0. calculate $h_{1 / 2}$ and $h_{N+1 / 2}$ using boundary formula

Step 1. $M=1 \quad$ ! looking for starting and ending points for smooth region using Lemma 1

$$
\begin{aligned}
& \text { start_point }(\mathrm{M})=1 \quad \text { ! smooth region starting point } \\
& \text { DO } \mathrm{i}=1, \mathrm{~N}-1 \\
& \quad \text { calculate } \beta_{0}, \beta_{1}, \beta_{2} \\
& \tau_{5}=\left|\beta_{0}-\beta_{2}\right| \\
& \text { IF } \tau_{5}>\min \left(\beta_{0}, \beta_{1}, \beta_{2}\right) \text { THEN } \\
& \quad \text { end_point }(\mathrm{M})=\mathrm{i} \quad \text { ! smooth region ending point } \\
& \quad \mathrm{M}=\mathrm{M}+1 \\
& \quad \text { start_point }(\mathrm{M})=\mathrm{i}+1 \\
& \quad \text { calculate } h_{i+1 / 2} \text { using WENO scheme: }
\end{aligned}
$$

$$
h_{i+\frac{1}{2}}=\sum_{k=0}^{2} \omega_{k} \hat{f}^{k}\left(x_{i+\frac{1}{2}}\right)
$$

END IF

END DO

end_point $(\mathrm{M})=\mathrm{N}$

Step 2. DO $\mathrm{k}=1, \mathrm{M} \quad$ ! calculate fluxes on smooth region $k$ using a high order scheme

$$
\begin{aligned}
& \text { DO } \mathrm{j}=\text { start_point }(\mathrm{k}), \text { end_point }(\mathrm{k})-1 \\
& \quad \text { calculate the right hand side } d_{i+1 / 2}
\end{aligned}
$$

END DO

solve the equations(general form)

$$
\alpha h_{i-1 / 2}+\gamma h_{i+1 / 2}+\beta h_{i+3 / 2}=d_{i+1 / 2}
$$

where, $i=$ start_point $(\mathrm{k}), \cdots$, end_point(k)-1.

\section{END DO}

\section{END}

In step 2, four different high order schemes controlled by the coefficients of $\alpha, \beta, \gamma$ and formulation $d_{i+1 / 2}$ are used,

(1) The fourth order central difference scheme

$$
\alpha=\beta=0, \gamma=1
$$

and

$$
d_{i+1 / 2}=\frac{1}{12}\left(-f_{i+2}+7 f_{i+1}+7 f_{i}-f_{i-1}\right)
$$


(2) The fifth order upstream difference scheme

$$
\alpha=\beta=0, \gamma=1
$$

and

$$
d_{i+1 / 2}=\frac{1}{60}\left(-3 f_{i+2}+27 f_{i+1}+47 f_{i}-13 f_{i-1}+2 f_{i-2}\right)
$$

(3) The 5th-order upwind compact scheme

$$
\alpha=\frac{2}{3}, \quad \beta=0, \gamma=1
$$

and

$$
d_{i+1 / 2}=\frac{1}{36}\left(-f_{i+2}+11 f_{i+1}+47 f_{i}+3 f_{i-1}\right)
$$

(4) The 6th-order Pade compact scheme

$$
\alpha=\beta=\frac{1}{3}, \gamma=1
$$

and

$$
d_{i+1 / 2}=\frac{1}{36}\left(f_{i+2}+29 f_{i+1}+29 f_{i}+f_{i-1}\right)
$$

For the 5th-order upstream difference scheme and the 5th-order upwind compact scheme, where the numerical flux is $h_{i+1 / 2}=h_{i+1 / 2}^{+}+h_{i+1 / 2}^{-}$. Only the positive part $h_{i+1 / 2}^{+}$is described above and the superscript " + " is dropped for simplicity. The $h_{i+1 / 2}^{-}$is evaluated following the symmetric rule about $x_{i+1 / 2}$.

Different formula can be used to calculate the computational domain boundary fluxes $h_{1 / 2}^{ \pm}$and $h_{N+1 / 2}^{ \pm}$. For example, the biased sixth order formula are used in this paper to calculate the boundary fluxes $h_{1 / 2}^{ \pm}$ and $h_{N+1 / 2}^{ \pm}$,

$$
\left\{\begin{array}{l}
h_{1 / 2}^{ \pm}=\frac{1}{60}\left(-2 f_{-1}^{ \pm}+22 f_{0}^{ \pm}+57 f_{1}^{ \pm}-23 f_{3}^{ \pm}+7 f_{4}^{ \pm}-f_{5}^{ \pm}\right) \\
h_{N+1 / 2}^{ \pm}=\frac{1}{60}\left(-f_{N-3}^{ \pm}+7 f_{N-2}^{ \pm}-23 f_{N-1}^{ \pm}+57 f_{N}^{ \pm}+22 f_{N+1}^{ \pm}-2 f_{N+2}^{ \pm}\right)
\end{array}\right.
$$

\section{$3 \quad$ Numerical Examples}

For the examples of sections 3.1-3.5, the 4th order Runge-Kutta-type method[40] is used for the time marching of the governing equations.

\subsection{Linear transport equation}

The linear transport equation is used to test the accuracy of the new scheme.

$$
\begin{gathered}
\frac{\partial u}{\partial t}+\frac{\partial u}{\partial x}=0,-1<x<1 \\
u(x, 0)=u_{0}(x), \text { periodic }
\end{gathered}
$$

(1)

$$
u_{0}(x)=\sin (2 \pi x)
$$


Table 2: Accuracy on $u_{t}+u_{x}=0$ with $u_{0}(x)=\sin (2 \pi x), \mathrm{t}=1$

\begin{tabular}{|c|c|c|c|c|c|}
\hline Scheme & $N$ & $L_{\infty}$ error & $L_{\infty}$ order & $L_{1}$ error & $L_{1}$ order \\
\hline \multirow{5}{*}{ 4-central } & 20 & $0.4335 \mathrm{e}-1$ & - & $0.2346 \mathrm{e}-1$ & - \\
& 40 & $0.2960 \mathrm{e}-2$ & 3.872 & $0.1544 \mathrm{e}-2$ & 3.925 \\
& 80 & $0.1911 \mathrm{e}-3$ & 3.953 & $0.9629 \mathrm{e}-4$ & 4.003 \\
& 160 & $0.1205 \mathrm{e}-4$ & 3.987 & $0.5917 \mathrm{e}-5$ & 4.024 \\
& 320 & $0.7473 \mathrm{e}-6$ & 4.011 & $0.3644 \mathrm{e}-6$ & 4.021 \\
\hline \multirow{5}{*}{ 5-upstream } & 20 & $0.1051 \mathrm{e}-1$ & - & $0.6068 \mathrm{e}-2$ & - \\
& 40 & $0.3625 \mathrm{e}-3$ & 4.858 & $0.2095 \mathrm{e}-3$ & 4.856 \\
& 80 & $0.1142 \mathrm{e}-4$ & 4.988 & $0.6692 \mathrm{e}-5$ & 4.968 \\
& 160 & $0.3533 \mathrm{e}-6$ & 5.015 & $0.2101 \mathrm{e}-6$ & 4.993 \\
& 320 & $0.1095 \mathrm{e}-7$ & 5.012 & $0.6573 \mathrm{e}-8$ & 4.998 \\
\hline \multirow{5}{*}{ 5-upwind } & 20 & $0.2900 \mathrm{e}-2$ & - & $0.1496 \mathrm{e}-2$ & - \\
& 40 & $0.7107 \mathrm{e}-4$ & 5.351 & $0.4185 \mathrm{e}-4$ & 5.160 \\
& 80 & $0.2213 \mathrm{e}-5$ & 5.005 & $0.1325 \mathrm{e}-5$ & 4.981 \\
& 160 & $0.7221 \mathrm{e}-7$ & 4.938 & $0.4263 \mathrm{e}-7$ & 4.958 \\
& 320 & $0.2328 \mathrm{e}-8$ & 4.955 & $0.1359 \mathrm{e}-8$ & 4.971 \\
\hline \multirow{5}{*}{ 6-Pade } & 20 & $0.5781 \mathrm{e}-2$ & - & $0.1525 \mathrm{e}-2$ & - \\
& 40 & $0.1330 \mathrm{e}-3$ & 5.442 & $0.4057 \mathrm{e}-4$ & 5.232 \\
& 80 & $0.2446 \mathrm{e}-5$ & 5.765 & $0.7561 \mathrm{e}-6$ & 5.746 \\
& 160 & $0.4242 \mathrm{e}-7$ & 5.850 & $0.1189 \mathrm{e}-7$ & 5.991 \\
& 320 & $0.6903 \mathrm{e}-9$ & 5.941 & $0.1818 \mathrm{e}-9$ & 6.031 \\
\hline
\end{tabular}

Table 2 gives the error and accuracy. It can be seen that every scheme obtains its expected accuracy. This shows the discontinuity detector does not affect the accuracy of the smooth solution.

(2)

$$
u_{0}(x)= \begin{cases}-x \sin \left(3 \pi x^{2} / 2\right), & -1 \leq x<-1 / 3 \\ |\sin (2 \pi x)|, & -1 / 3 \leq x \leq 1 / 3 \\ 2 x-1-\sin (3 \pi x) / 6, & \text { otherwise }\end{cases}
$$

Fig. 2 shows the numerical solutions at $t=8$ with 200 grid points. It can be seen that the 5 th-order upwind compact scheme is nearly as good as the 6th-order Pade scheme, and the 4th-order central scheme deviates slightly from the exact solution at two extremum points.

(3)

$$
u_{0}(x)= \begin{cases}\frac{1}{6}(G(x, \beta, z-\delta)+G(x, \beta, z+\delta)+4 G(x, \beta, z)), & -0.8 \leq x \leq-0.6 \\ 1, & -0.4 \leq x \leq-0.2 \\ 1-|10(x-0.1)|, & 0 \leq x \leq 0.2 \\ \frac{1}{6}(F(x, \alpha, \alpha-\delta)+F(x, \alpha, \alpha+\delta)+4 F(x, \alpha, a)), & 0.4 \leq x \leq 0.6 \\ 0, & \text { otherwise }\end{cases}
$$

Same as in Ref.[32], the constants for this case are taken as $a=0.5, z=-0.7, \delta=0.005, \alpha=10$, and $\beta=\log 2 / 36 \delta^{2}$. The solution contains a smooth combination of Gaussians, a square wave, a sharp triangle wave, and a half ellipse.

The results at $t=8$ with 200 grid points are shown in Figs. 3 and 4 . It can be seen that, the 6th-order Pade scheme resolve the Gaussians, the square wave and the triangle wave best, but it overshoots at the extremum point of the ellipse wave. The 5th-order upwind compact scheme is more accurate than the 5th-order upstream central scheme, and the latter is better than the 4th-order central scheme. 


\subsection{Nonlinear transport equation}

The nonlinear transport equation is used as the fourth test case and can be written as

$$
\begin{gathered}
\frac{\partial u}{\partial t}+u \frac{\partial u}{\partial x}=0, \quad 0 \leq x \leq 2 \pi \\
u(x, 0)=0.3+0.7 \sin x, \quad 0 \leq x \leq 2 \pi, \text { with a periodic boundary condition }
\end{gathered}
$$

The Lax-Friedrichs splitting method is used, in which $f^{ \pm}(u)=\frac{1}{2}(f(u) \pm a u)$ and $a=\max u f^{\prime}(u) \mid$. Fig. 5 shows the results at $t=2$ with the grid number of $N=80$. For this case, there is indistinguishable difference between all the schemes tested in this paper.

\subsection{Viscous Burgers equation}

The viscous Burgers equation is the fifth test case, and is written as

$$
\begin{gathered}
\frac{\partial u}{\partial t}+u \frac{\partial u}{\partial x}=\frac{1}{R e} \frac{\partial^{2} u}{\partial x^{2}}, \quad a \leq x \leq b \\
u(a)=\tanh (-a \operatorname{Re} / 2), \quad u(b)=\tanh (-b \operatorname{Re} / 2)
\end{gathered}
$$

The steady state solution of Eq. (33) with boundary condition $(34)$ is $u(x)=\tanh (-x R e / 2)$. At $x=0$, the shock is formed with a large Re number. In our computation, $a=-1, b=1$ and $R e=1000$ are used. The results are shown in Fig. 6. For this case, the iteration steps and the final solutions are exactly the same for all the tested schemes.

\subsection{D Shock Wave Tube, Shu-Osher Problem}

This problem is governed by the one-dimensional Euler equations with following initial condition:

$$
(\rho, u, p)= \begin{cases}(3.857143,2.629369,10.3333), & \text { when } x<-4 \\ (1+\varepsilon \sin (5 x), 0.0,1.0), & \text { when } x \geq-4 .\end{cases}
$$

where, $\varepsilon=0.2$. This case represents a Mach 3 shock wave interacting with a sine entropy wave[41]. The Steger-Warming flux vector splitting method[42] is used to calculate the interface fluxes. The results at time $t=1.8$ with mesh size of 300 are plotted in Fig. 7. The "exact" solutions are the numerical solutions of the original WENO-Z scheme with grid points of $N=4000$. It can be seen that, 6 -Pade scheme obtains highest maximum and lowest minimum, 5-upwind scheme is the next one. This cases shows, for the complex smooth solution, higher order scheme can obtain more accurate solution.

\subsection{Two-dimensional Shock/Shear Layer Interaction}

A two-dimensional shock/shear layer interaction problem is solved to demonstrate these schemes for multidimensional flows. The two-dimensional Navier-Stokes equations are solved for this problem:

$$
\frac{\partial \mathbf{U}}{\partial t}+\frac{\partial \mathbf{E}}{\partial x}+\frac{\partial \mathbf{F}}{\partial y}=\frac{1}{R e}\left[\frac{\partial \mathbf{E}_{\mathbf{v}}}{\partial x}+\frac{\partial \mathbf{F}_{\mathbf{v}}}{\partial y}\right]
$$

where 


$$
\mathbf{U}=\left[\begin{array}{l}
\rho \\
\rho u \\
\rho v \\
\rho e
\end{array}\right], \mathbf{E}=\left[\begin{array}{l}
\rho u \\
\rho u^{2}+p \\
\rho u v \\
u(\rho e+p)
\end{array}\right], \mathbf{F}=\left[\begin{array}{l}
\rho v \\
\rho u v \\
\rho v^{2}+p \\
v(\rho e+p)
\end{array}\right], \mathbf{E}_{\mathbf{v}}=\left[\begin{array}{l}
0 \\
\tau_{x x} \\
\tau_{x y} \\
u \tau_{x x}+v \tau_{x y}+q_{x}
\end{array}\right], \mathbf{F}=\left[\begin{array}{l}
0 \\
\tau_{x y} \\
\tau_{y y} \\
u \tau_{x y}+v \tau_{y y}+q_{y}
\end{array}\right],
$$

where $\tau_{x x}=\mu\left(\frac{4}{3} \frac{\partial u}{\partial x}-\frac{2}{3} \frac{\partial v}{\partial y}\right), \tau_{x y}=\mu\left(\frac{\partial v}{\partial x}+\frac{\partial u}{\partial y}\right), \tau_{x y}=\mu\left(\frac{4}{3} \frac{\partial v}{\partial y}-\frac{2}{3} \frac{\partial u}{\partial x}\right)$.

$$
p=(\gamma-1)\left(\rho e-\rho\left(u^{2}+v^{2}\right) / 2\right), \gamma=1.4 \text {. }
$$

An oblique shock with angle $\beta=12^{\circ}$ is made to impact on a spatially developing mixing layer at an initial convective Mach number of 0.5 . The computation domain is taken to be $[x, y]=[0,200] \times[-20,20]$. The inflow is specified with a hyperbolic tangent profile,

$$
u=2.5+0.5 \tanh (2 y)
$$

For the upper stream inflow, $\rho_{u}=1.6374, p_{u}=0.3327$; for the lower stream inflow, $\rho_{l}=0.3626, p_{l}=0.3327$. The upper boundary condition is taken from the flow properties behind the oblique shock. The lower wall uses a slip condition.

Fluctuations are added to the v-component of velocity of the inflow as

$$
v^{\prime}=\sum_{k=1}^{2} a_{k} \cos \left(2 \pi k t / T+\phi_{k}\right) \exp \left(-y^{2} / b\right)
$$

with period $T=\lambda / u_{c}$, wavelength $\lambda=30$, convective velocity $u_{c}=2.68, b=10, a_{1}=a_{2}=0.05, \phi_{1}=0$ and $\phi_{2}=\pi / 2$.

The Prandtl number is set to 0.72 , and the Reynolds number is chosen to be 500 . The viscosity $\mu$ is calculated by using Sutherland formula. The 4th-order central difference scheme[43] is used for the viscous terms. The uniform grid, $321 \times 81$, is used. The time step is taken as follows[16]:

$$
\Delta t=\delta \frac{\Delta t_{x} \Delta t_{y}}{\Delta t_{x}+\Delta t_{y}}, \text { with } \Delta t_{x}=\frac{\Delta x}{\max _{i, j}\left(\left|u_{i, j}\right|+c_{i, j}\right)}, \Delta t_{y}=\frac{\Delta y}{\max _{i, j}\left(\left|v_{i, j}\right|+c_{i, j}\right)}
$$

where $\delta=0.5$ is the CFL number.

The Steger-Warming flux vector splitting method[42] is used to calculate the interface fluxes. The density contours are shown in Fig. 8. It can be seen that all the schemes resolve the shock wave and vortex structures very well. The 6th-order Pade scheme gives the most clear vortex structures.

\subsection{Transonic Converging-Diverging Nozzle}

In order to compare the behavior of different schemes for the applications, the Roe's flux difference scheme[44] is used as the Riemann solver with the reconstruction of different schemes. The unfactored implicit Gauss-Seidel line relaxation method[45] is adopted for the time marching.

First, the steady transonic flow of a converging-diverging nozzle is calculated. The nozzle was designed and tested at NASA and was named as Nozzle A1[46] and is symmetric about the centerline. Hence only the upper half of nozzle is computed. The mesh size is $175 \times 50$. The grid is clustered near the wall. The inlet Mach number is 0.22 . This case is calculated as inviscid flow.

Fig. 9 is the computed pressure contour, which shows that two shocks emanating from the walls downstream of the throat, interacting each other and reflecting on the walls. From the convergence histories shown in Fig. 10, it can be seen that the rank of schemes is 5-upstream, 4-central, 5-upwind, and 6-Pade. Fig. 11 shows the pressure coefficients at the upper wall. It is observed that all four schemes generate a slight oscillation in the region behind the reflected shocks. The convergence may be affected by this oscillation. 


\subsection{Transonic RAE2822 Airfoil}

The transonic RAE2822 airfoil is the second example of the steady flow. For this case, the Reynolds averaged Navier-Stokes equation with the Baldwin-Lomax turbulent model are solved. The computational region is divided into two blocks, and the mesh size of $128 \times 55$ is used for each block, the freestream Mach number $M_{\infty}$ is 0.729 , the Reynolds number based on chord length is $6.5 \times 10^{6}$, and the angle of attack is $2.31^{\circ}$.

Fig. 12 is the pressure contours calculated by 6-Pade scheme, it shows the shock wave captured well. Fig. 13 gives the comparison of convergence histories. Again, the rank is still 5-upstream, 4-central, 5upwind, and 6-Pade. Fig. 14 shows that there is few difference on the pressure coefficients at the airfoil surface.

\section{Conclusions}

In this paper, several high order schemes are combined with the fifth weighted essentially nonoscillatory(WENO) by using the discontinuity detector proposed by Shen and Zha[38]. Numerical examples show that

(1) The parameter-free discontinuity detector is effective for constructing the high order hybrid schemes.

(2) For a complex smooth solution, the higher order scheme can obtain more accurate solution. At a same stencil, the highest order scheme is the compact scheme. For example,the optimal scheme is the 6th-order Pade scheme if a five-points stencil is used.

(3) For a simple steady flow, the difference between different schemes can be neglected. But for convergence, the upwind biased scheme is prior to the central scheme. For example, the 5th-order upstream scheme is better than the 4th-order central scheme, the 5th-order upwind compact scheme is better than the 6th-order Pade scheme.

(4) How to improve the convergence of a hybrid scheme is still an open issue.

\section{References}

[1] Chi-Wang Shu, "High Order Weighted Essentially Nonoscillatory Schemes for Convection Dominated Problems," SIAM Review, vol. 51, pp. 82-126, 2009.

[2] Shuhai Zhang, Shufen Jiang, Chi-Wang Shu, "Development of nonlinear weighted compact schemes with increasingly higher order accuracy," Journal of Computational Physics, vol. 227, pp. 7294-7321, 2008 .

[3] D.S. Balsara, C.-W. Shu, "Monotonicity preserving weighted essentially non-oscillatory schemes with increasingly high order of accuracy," J.Comput.Phys., vol. 160, pp. 405-452, 2000.

[4] C. Canuto, M. Y. Hussaini, A. Quarteroni, T. A. Zang, Spectral Methods in Fluid Dynamics. Springer, New York, 1987.

[5] S. A. Lele, "Compact finite difference schemes with spectral-like resolution," Journal of Computational Physics, vol. 103, No. 1, pp. 16-42, 1992.

[6] Santhanam Nagarajan, Sanjiva K. Lele, Joel H. Ferziger, "A robust high-order compact method for large eddy simulation," Journal of Computational Physics, vol. 191, pp. 392-419, 2003. 
[7] Noma Park, Jung Yul Yoo, Haecheon Choi , "Discretization errors in large eddy simulation: on the suitability of centered and upwind-biased compact difference schemes ," J.Comput.Phys., vol. 198, pp. 580-616, 2004.

[8] M. Piller, E. Stalio, "Finite-volume compact schemes on staggered grids," Journal of Computational Physics, vol. 197, pp. 299-340, 2004.

[9] Ali Uzun, Gregory A. Blaisdell, Anastasios S. Lyrintzis, "Application of Compact Schemes to Large Eddy Simulation of Turbulent Jets," Journal of Scientific Computing, vol. 21, pp. 283-319, 2004.

[10] Chaoqun Liu, "High performance computation for DNS/LES," Applied Mathematical Modelling, vol. 30, pp. 1143-1165, 2006.

[11] Donald P. Rizzetta, Miguel R. Visbal, Philip E. Morgan, "A high-order compact finite-difference scheme for large-eddy simulation of active flow control," Progress in Aerospace Sciences, vol. 44, pp. 397-426, 2008.

[12] B. Cockburn, C.W. Shu, "Nonlinearly stable compact schemes for shock calculations," SIAM Journal on Numerical Analysis, vol. 31, pp. 607-627, 1994.

[13] K. S. Ravichandran, "Higher Order KFVS Algorithms Using Compact Upwind Difference Operators," Journal of Computational Physics, vol. 130, pp. 161-173, 1997.

[14] Guo-Hua Tu, Xiang-Jiang Yuan, "A characteristic-based shock-capturing scheme for hyperbolic problems," Journal of Computational Physics, vol. 225, pp. 2083-2097, 2007.

[15] N. A. Adams, K. Shariff, "A High-Resolution Hybrid Compact-ENO Scheme for Shock-Turbulence Interaction Problems," Journal of Computational Physics, vol. 127, pp. 27-51, 1996.

[16] S. Pirozzoli, "Conservative hybrid compact-WENO schemes for shock-turbulence interaction," J.Comput.Phys., vol. 178, pp. 81-117, 2002.

[17] Yu-Xin Ren, Miao'er Liu, Hanxin Zhang, "A characteristic-wise hybrid compact-WENO scheme for solving hyperbolic conservation laws," Journal of Computational Physics, vol. 192, pp. 365-386, 2003.

[18] Qiang Zhou, Zhaohui Yao, Feng He, M.Y. Shen, "A new family of high-order compact upwind difference schemes with good spectral resolution," Journal of Computational Physics, vol. 227, pp. 13061339, 2007.

[19] Y.-Q. Shen, G.-W. Yang, Z. Gao, "High-resolution finite compact difference schemes for hyperbolic conservation laws," J.Comput.Phys., vol. 216, pp. 114-137, 2006.

[20] Y.-Q. Shen, G.-W. Yang, "Hybrid finite compact-WENO schemes for shock calculation," International Journal for Numerical Methods in Fluids, vol. 53, pp. 531-560, 2007.

[21] Xiaogang Deng, Hanxin Zhang, "Developing High-Order Weighted Compact Nonlinear Schemes," Journal of Computational Physics, vol. 165, pp. 22-44, 2000.

[22] H. C. Yee, Sjogreen, B., "Adaptive filtering and limiting in compact high order methods for multiscale gas dynamics and MHD systems," Computers $\&$ Fluids, vol. 37, pp. 593-619, 2008.

[23] M. R. Visbal, D. Gaitonde, "High Order-Accurate Methods for Complex Unsteady Subsonic Flows," AIA A Journal, vol. 37, No. 10, pp. 1231-1239, 1999.

[24] A.W. Cook, W.H. Cabot, "A high-wavenumber viscosity for high resolution numerical method," Journal of Computational Physics, vol. 195, pp. 594-601, 2004. 
[25] A.W. Cook, W.H. Cabot, "Hyperviscosity for shock-turbulence interactions," Journal of Computational Physics, vol. 203, pp. 379-385, 2005.

[26] B. Fiorina, S.K. Lele, "An artificial nonlinear diffusivity method for supersonic reacting flows with shocks," Journal of Computational Physics, vol. 222, pp. 246-264, 2007.

[27] S. Kawai, S.K. Lele, "Localized artificial diffusivity scheme for discontinuity capturing on curvilinear meshes," Journal of Computational Physics, vol. 227, pp. 9498-9526, 2008.

[28] C. Bogey, N. de Cacqueray, C. Bailly, "A shock-capturing methodology based on adaptative spatial filtering for high-order non-linear computations," Journal of Computational Physics, vol. 228, pp. 1447-1465, 2009.

[29] H. C. Yee, N. D. Sandham, M. J. Djomehri, "Low-Dissipative High-Order Shock-Capturing Methods Using Characteristic-Based Filters," Journal of Computational Physics, vol. 150, pp. 199-238, 1999.

[30] S.-C. Lo, G. A. Blaisdell, A. S. Lyrintzis, "High-order shock capturing schemes for turbulence calculations," International Journal For Numerical Methods in Fluids, vol. 62, pp. 473-498, 2010.

[31] X.D. Liu, S. Osher, T. Chan, "Weighted essentially non-oscillatory schemes," J.Comput.Phys., vol. 115 , pp. 200-212, 1994.

[32] G.-S. Jiang, C.-W. Shu, "Efficient implementation of weighted ENO schemes," J.Comput.Phys., vol. 126, pp. 202-228, 1996.

[33] Y.-Q. Shen, G.-Z. Zha, "Improvement of the WENO scheme smoothness estimator," International Journal for Numerical Methods in Fluids, vol. 64, pp. 653-675, 2010.

[34] Y.-Q. Shen, G.-C. Zha, B.-Y. Wang, "Improvement of stability and accuracy of implicit WENO scheme," AIAA Journal, vol. 47, pp. 331-344, 2009.

[35] A.K. Henrick, T.D. Aslam, J.M. Powers, "Mapped weighted essentially non-oscillatory schemes:Achiving optimal order near critical points," J.Comput.Phys., vol. 208, pp. 206-227, 2005.

[36] R. Borges, M. Carmona, B. Costa, W.S. Don, "An improved weighted essentially non-oscillatory scheme for hyperbolic conservation laws," Journal of Computational Physics, vol. 227, pp. 3191-3211, 2008 .

[37] Y.-Q. Shen, G.-C. Zha, "Improvement of weighted essentially non-oscillatory schemes near discontinuity." AIAA paper 2009-3655, 2009.

[38] Y.-Q Shen, G.-C Zha, "Generalized finite compact difference scheme for shock/complex flowfield interaction," Journal of Computational Physics, vol. 230, pp. 4419-4436, 2011.

[39] Y.-Q. Shen, R.-Q. Wang, H.-Z. Liao, " A fifth-order accurate weighted ENN difference scheme and its applications," Journal of Computational Mathematics, vol. 19, pp. 531-538, 2001.

[40] C.-W. Shu, O. Osher, "Efficient implementation of essentially non-oscillatory shock capturing schemes," Journal of Computational Physics, vol. 77, pp. 439-471, 1988.

[41] C.-W. Shu, O. Osher, "Efficient Implementation of Essentially Non-Oscillatory Shock Capturing Schemes, II," Journal of Computational Physics, vol. 83, pp. 32-78, 1989.

[42] J. L. Steger, R. F. Warming, "Flux vector splitting of the inviscid gasdynamic equations with application to finite-difference methods," Journal of Computational Physics, vol. 40, pp. 263-293, 1981. 
[43] Y.-Q. Shen, G.-Z. Zha, X.-Y. Chen, "High order conservative differencing for viscous terms and the application to vortex-induced vibration flows," Journal of Computational Physics, vol. 228, pp. 82838300, 2009.

[44] P. Roe, "Approximate Riemann solvers, parameter vectors, and difference schemes," Journal of Computational Physics, vol. 43, pp. 357-372, 1981.

[45] Y.-Q. Shen, B.-Y. Wang, G.-C. Zha, "Comparison study of implicit Gauss-Seidel line iteration method for transonic flows." AIAA-2007-4332, June 2007.

[46] Mason, M. L. and Putnam, L. E. , "The Effect of Throat Contouring on Two-Dimensional ConvergingDiverging Nozzles at Static Conditions ." NASA Technical Paper 1704, 1980. 


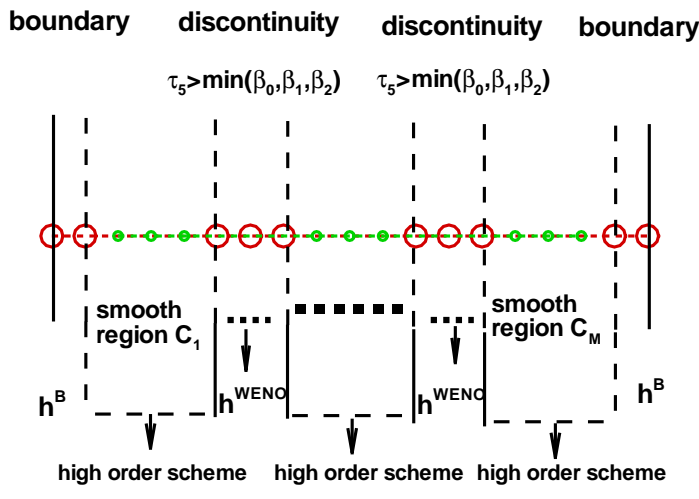

Figure 1: The sketch of the hybrid schemes

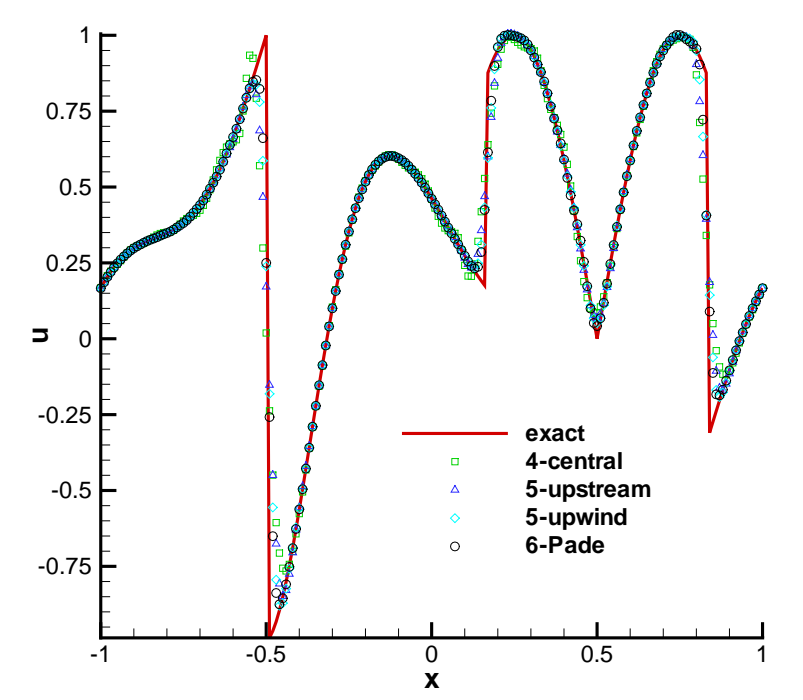

Figure 2: Numerical results, $\mathrm{t}=8$

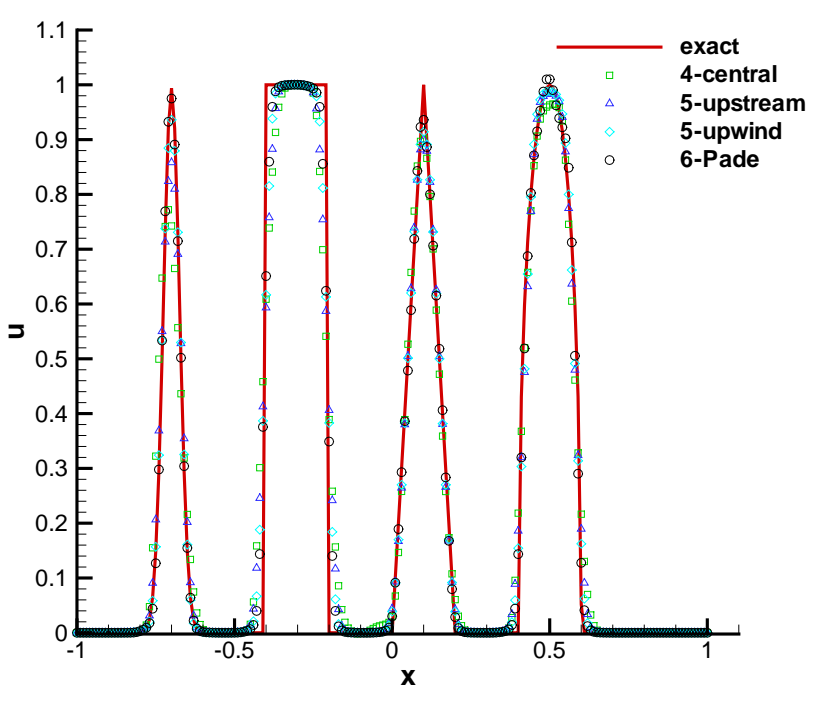

Figure 3: Numerical results, $\mathrm{t}=8$

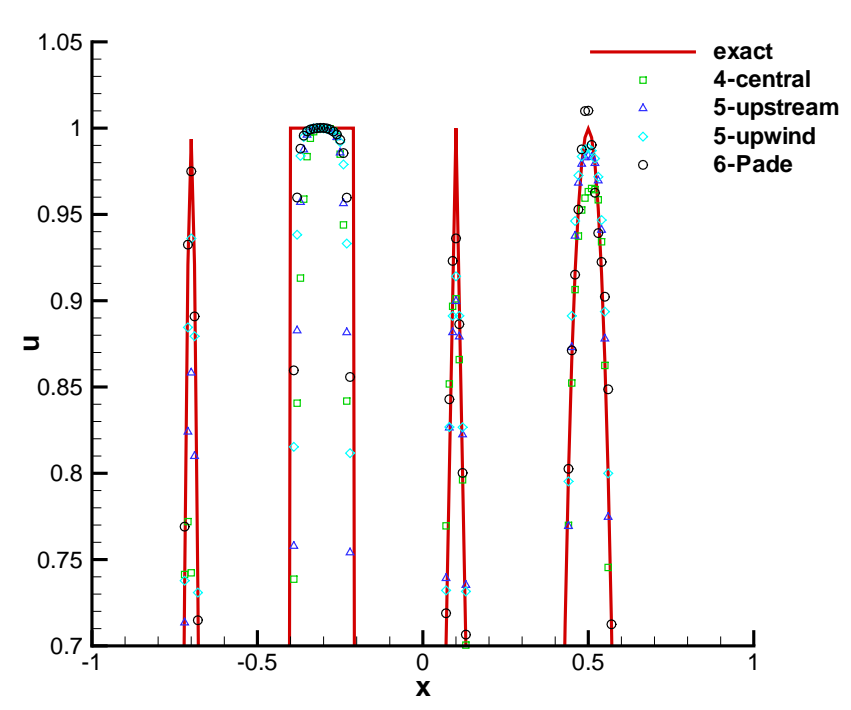

Figure 4: Locally enlarged plot of Fig. 3 


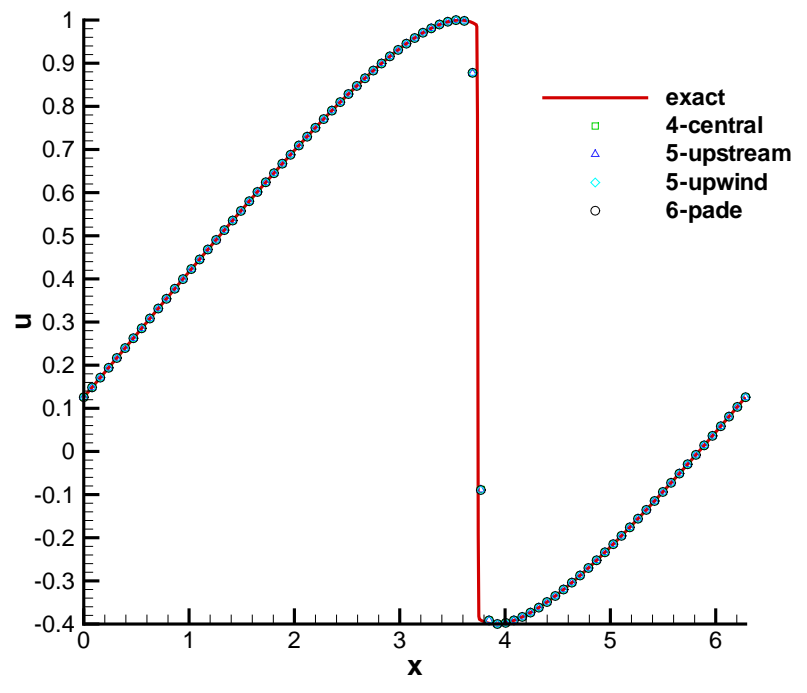

Figure 5: Nonlinear transport equation, $\mathrm{t}=2$

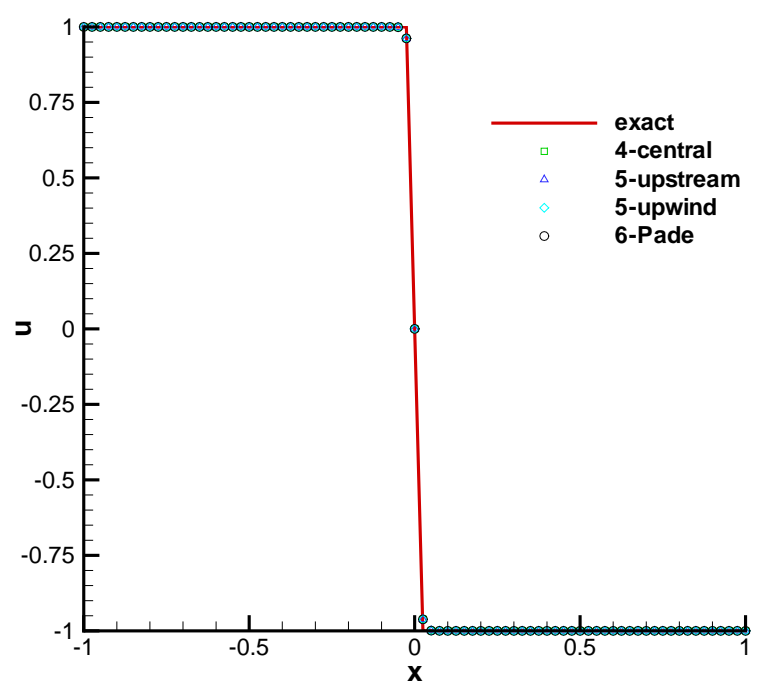

Figure 6: Viscous Burgers equation

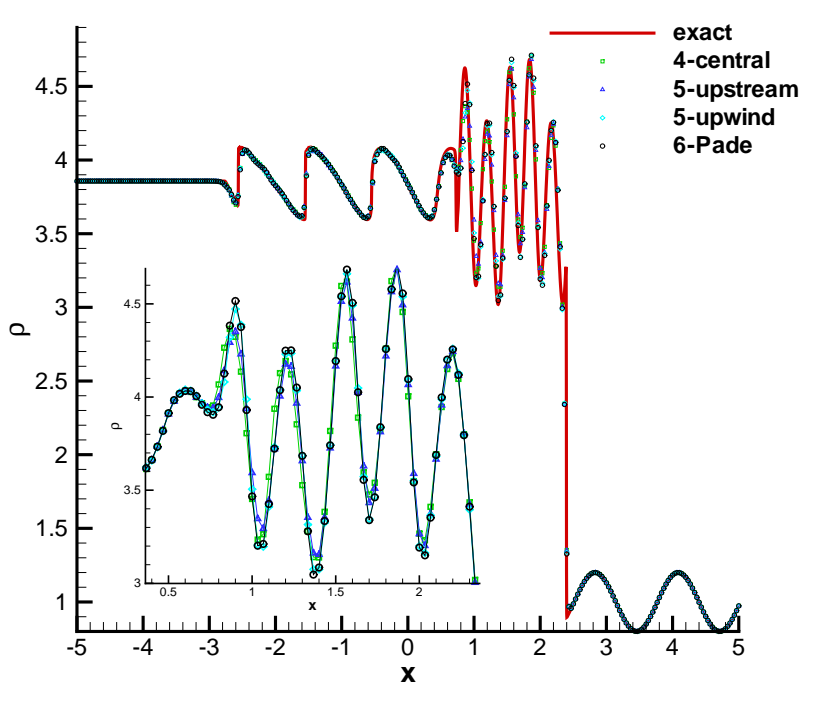

Figure 7: Shu-Osher problem

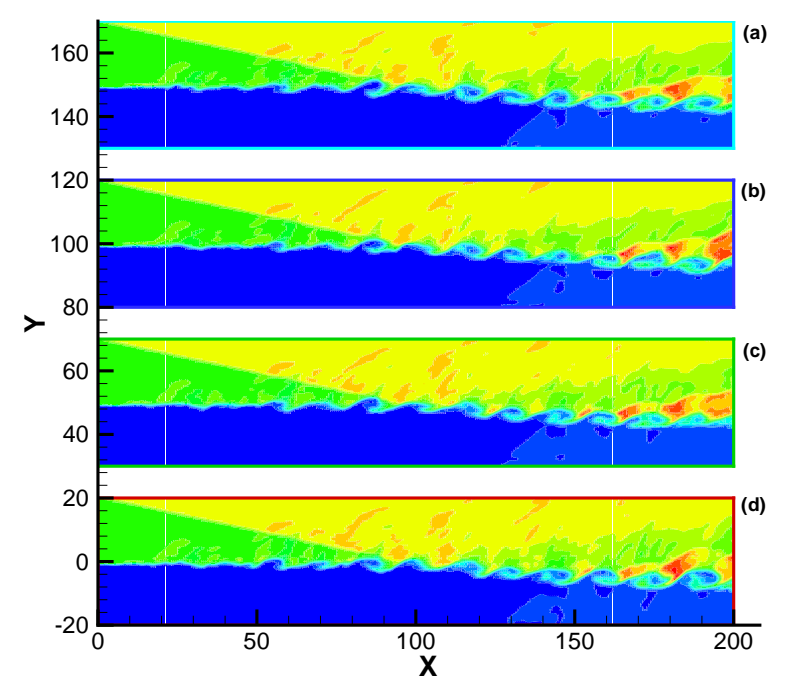

Figure 8: Density contours of the shock wave/shear interaction, $t=120$. (a) the 4 th-order central difference scheme, (b) the 5th-order upstream central scheme, (c) the 5th-order upwind compact scheme, (d) the 6th-order Pade scheme 


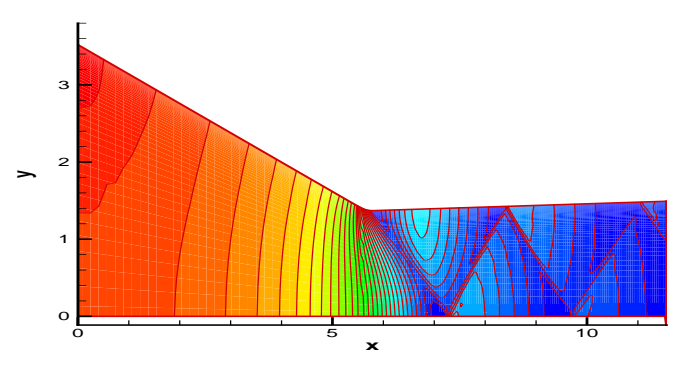

Figure 9: Pressure contours of the transonic converging-diverging nozzle flow

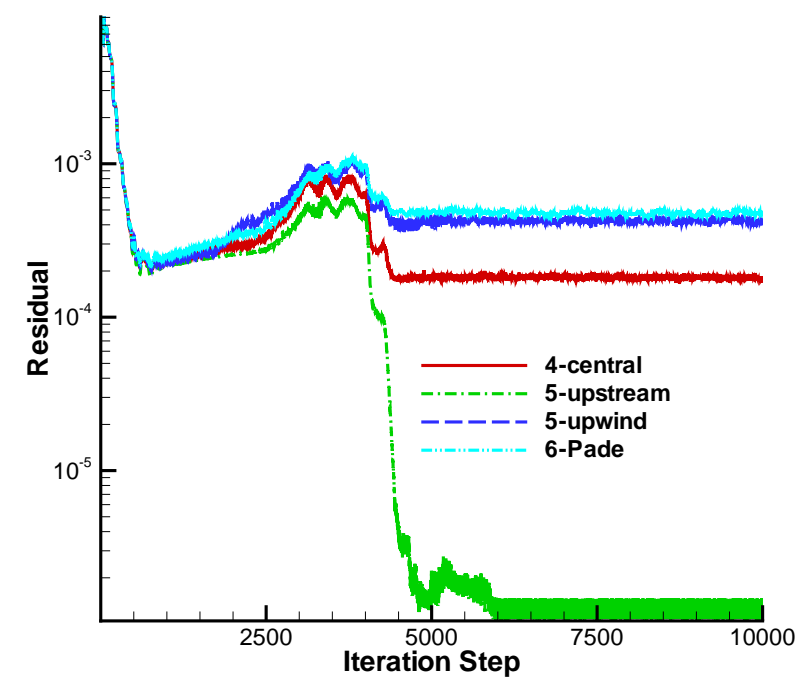

Figure 10: Convergence history of the transonic converging-diverging nozzle flow

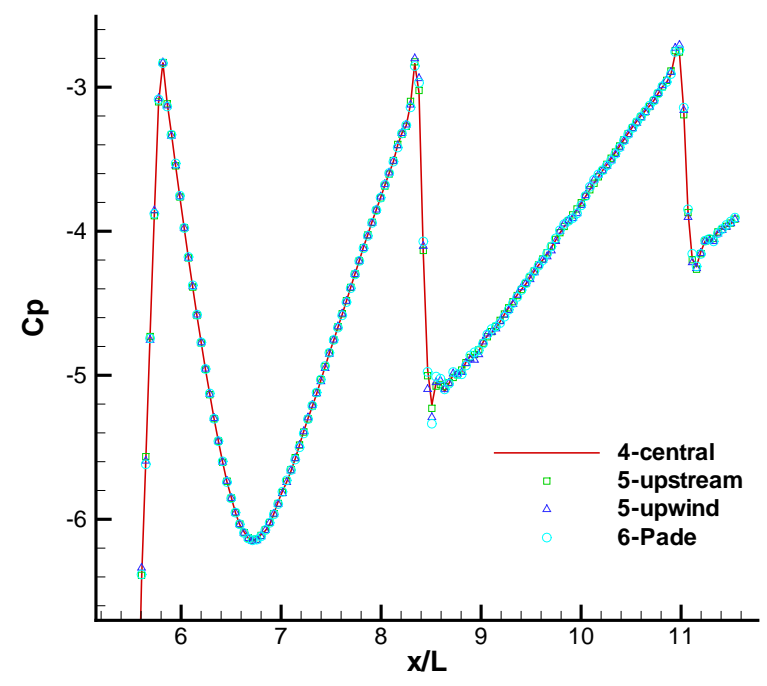

Figure 11: Pressure coefficients of the transonic converging-diverging nozzle flow

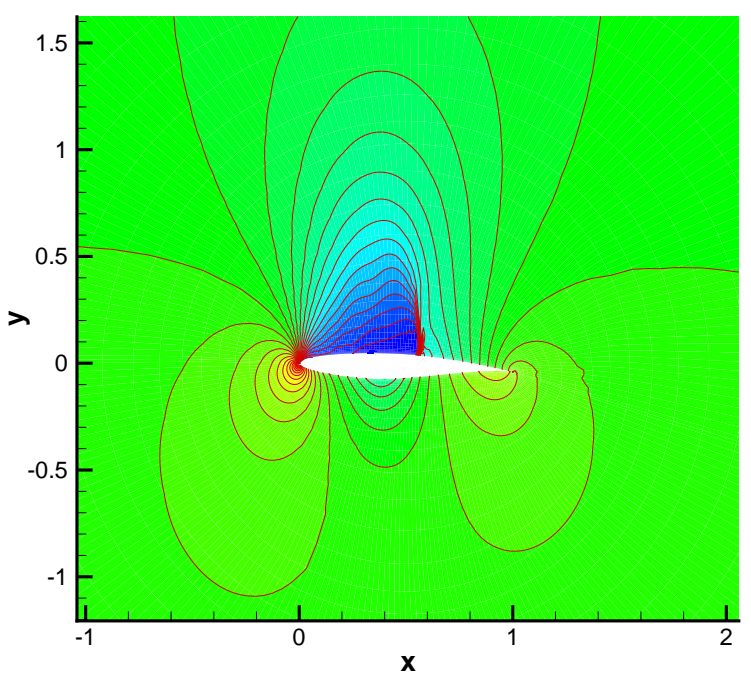

Figure 12: Pressure contours of the transonic flow for RAE2822 airfoil 


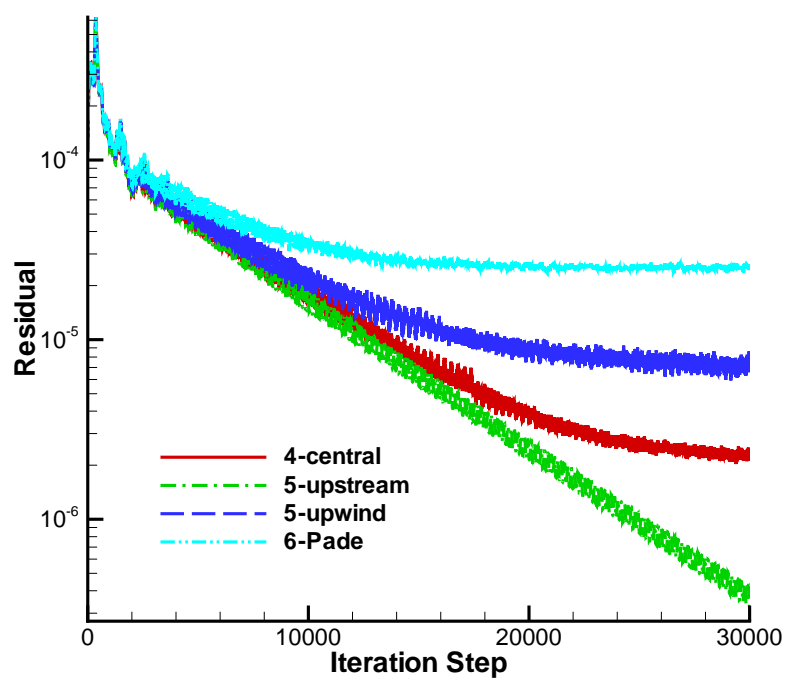

Figure 13: Convergence history of the transonic flow for RAE2822 airfoil

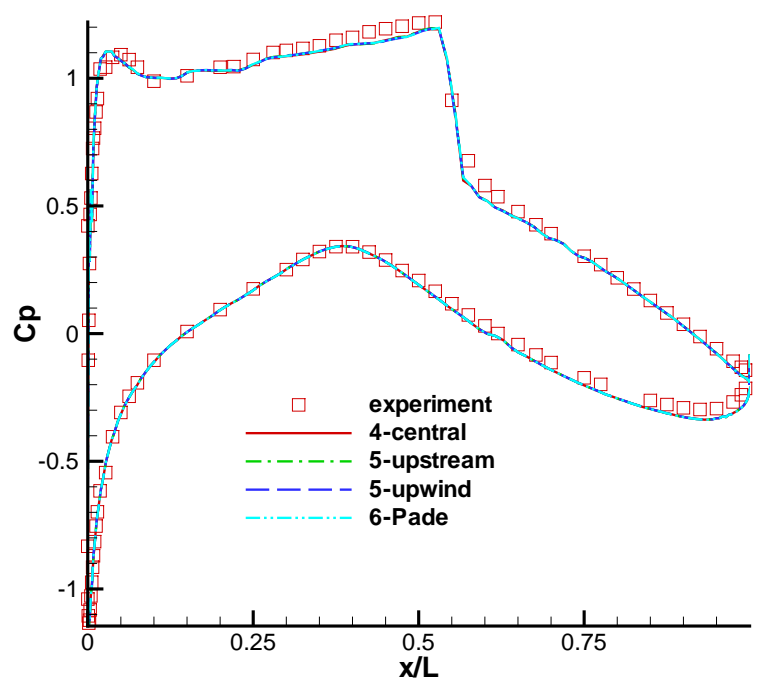

Figure 14: Pressure coefficients of the transonic flow for RAE2822 airfoil 